\title{
THEORETICAL ASPECTS ON THE MOVEMENT OF PLANT FRAGMENTS ON THE SURFACE OF A PLANE SIFTERS CLASSIFIER
}

\author{
AUGUSTINA PRUTEANU ${ }^{1 *}$, VALENTIN VLADUT ${ }^{1}$, LADISLAU DAVID $^{2}$ \\ ${ }^{I}$ National Institute of Research-Development for Machines and Installations Designed \\ to Agriculture and Food Industry - INMA, 6 Ion Ionescu de la Brad, Blv, sector 1, \\ Bucharest 71592, OP 18, Romania \\ ${ }^{2}$ University Politehnica of Bucharest, 313 Splaiul Independentei, sector 6, Bucharest \\ 060042, Romania
}

\begin{abstract}
This paper presents the main theoretical elements underlying the process of dimensional separation of the fragments of chopped medicinal plants. To sort chopped plant mixture from the same medicinal plant, there are used vibratory classifiers with plane sifters, which are the active working body. Case studies were conducted and there were analyzed, based on numerical applications, the movements of plant fragments on a classifier with linear vibrations along the sifter, existing in practice. Numerical simulation has provided information on trajectory parameters of the jump of a plant fragment on the vibratory plane sifter, useful elements in the theoretical grounding of the equipment workflow.
\end{abstract}

Keywords: plant fragment movement, plane sifter, vibratory classifier, trajectory

\section{INTRODUCTION}

The quality of herbal products obtained from the processing of medicinal plants depends on the quality of raw medicinal plants and the quality of primary and/or advanced processing products. The sequence of operations in primary processing of medicinal plants is mainly determined by raw material (species, plant organ, etc.) and the purpose of their exploitation (packaging and marketing in the form of teas, storage or further processing in various industries) [1].

Sorting is the operation of separation in several fractions $(3,4)$ according to certain criteria: size, weight, color, etc., of the components of a mixture of agricultural products found in the state of granules, fragments, particles, pieces, etc. (seeds, potato tubers, fruits, etc.). The purpose of this operation is to obtain uniform fractions in size, shape, weight, colour, etc., which can be used in further operations, achieving a high yield [2].

In practice, the sorting of inhomogeneous mixtures using plane sifters is achieved by using oscillatory or vibratory classifiers. The process is carried out by passing the mixtures that contain particles of various sizes over surfaces fitted with holes of certain shapes and sizes, respectively over plane or cylindrical sifters. The working process of the sifter comprises two stages: the first stage consists in the movement of mixture of plant fragments, spread in an even layer, and the second stage is the separation of plant fragments through the holes of the sifter due to the passing of fragments smaller than the sifters holes [3-5].

\footnotetext{
*Corresponding author, email:pruteanu_augustina@yahoo.com

(C) 2015 Alma Mater Publishing House
} 
At vibratory sifters, the oscillatory movement, characterised by frequency and amplitude of oscillations, occurs in a direction forming an angle $\alpha$ to the plane of the sifter [5-7].

Characteristic of these sorting surfaces is that particles movement on the sifter surface takes place in jumps [810]. This is advantageous, since the descendant trajectory of the particle approaches the normal to the surface of separation, facilitating the passage of particles through the holes [11-14].

The purpose of medicinal plants sorting is to obtain dimensional classes of upper and lower limits or welldefined particle sizes. This sorting is done mechanically by screening (sieving, sifting) by means of mechanical sifter equipment $[11,15]$.

Sifter working process comprises two stages: the first stage consists in the displacement of vegetal fragments matter, distributed in a uniform layer, and the second one consists in the separation of fragments through the sifter holes, because of the passing of fragments smaller than sifter holes size $[3,5,16]$.

Nowadays, we are noticing an increased interest in theoretical and experimental research of dimensional sorting devices in terms of working performances improving and for obtaining useful information in design activity.

Thus, in the paper was performed the numerical simulation of parameters of the trajectory of a vegetal fragment jump on the vibrating plane sifter, corresponding to real values of an equipment used in practice. Different parameters that influence the fragments movement have been varied for better understanding and quantify the researche effect on vegetal fragments trajectory.

The working principle of chopped plants sorter driven by two vibrating motors is shown in Figure 1. The equipment is used for separating food products: seeds, dried leaves, flowers, pulvis etc. [17].

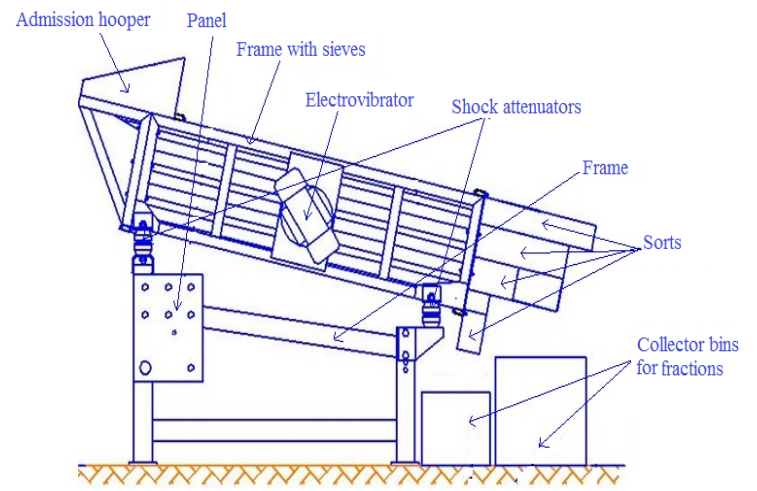

Fig. 1. Chopped plants sorter.

\section{THEORETICAL ASPECTS ON THE MOVELEMNT OF A PLANT FRAGMENT ON A VIBRATORY PLANE SIFTER WITH LONGITUDINAL MOVEMENT}

The kinematic regime of operation of plane sifters is very important and influences both the working capacity of plane sifters and the high quality of final product. The main technological parameter of the sieving process that correlates the vibration parameters (amplitude, frequency and acceleration) with its tilting, and determines the movement regime of the material on the sifter surface is the throwing coefficient $C[4,6,18]$.

\section{a) Movement of a plant fragment on a vibratory plane sifter}

The mathematical model corresponding to the movement of a plant fragment on an oscillatory plane sifter consists in identifying the forces and the directions of forces acting on a solid fragment found on an oscillatory surface.

Following are presented the equations of movement of a plant fragment on a plane oscillatory surface taking into account the two main directions of movement (Figure 2), downward (Figure 2 a) and upward (Figure 2 b). 
On a plant fragment moving downward on a plane oscillatory surface, acts the forces presented in Figure 2 a [7].

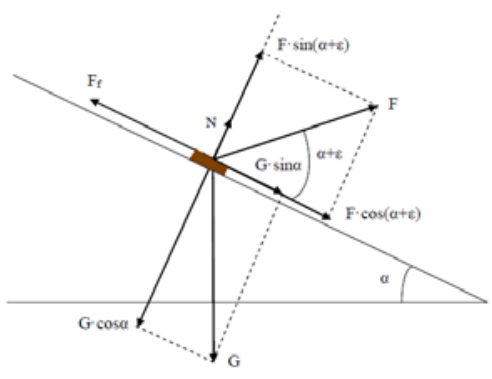

a)

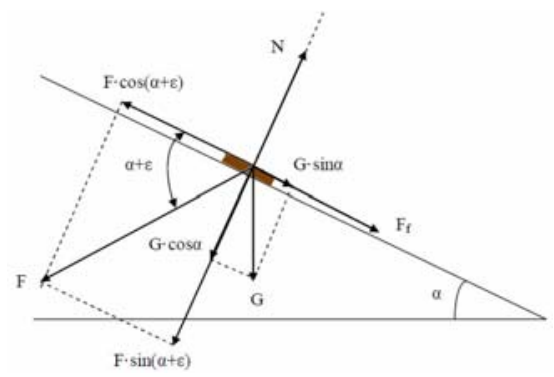

b)

Fig. 2. Plant fragment moving downward on a plane oscillatory surface, act the forces.

Equation of movement of the fragment is given by:

$$
m \cdot \frac{d v}{d t}=F \cdot \cos (\alpha+\varepsilon)+G \cdot \sin \alpha-F_{f}
$$

where $\mathrm{F}$, the inertial force, is:

$$
F=m \cdot a=\frac{G}{g} \cdot \omega^{2} \cdot r \cdot \cos \omega t
$$

and $\mathrm{N}$, reaction, is given by the equation:

$$
N=G \cdot \cos \alpha-F \cdot \sin (\alpha+\varepsilon)
$$

By replacing all terms in equation (1), is obtained:

$$
m \cdot a_{c}=F \cdot \cos (\alpha+\varepsilon)+G \cdot \sin \alpha-\operatorname{tg} \varphi \cdot[G \cdot \cos \alpha-F \cdot \sin (\alpha+\varepsilon)]
$$

For

$$
F=\frac{G}{g} \omega^{2} \cdot A \cdot \cos \omega t
$$

Thus:

$$
m \cdot a_{c}=\frac{G}{g} \cdot \omega^{2} \cdot A \cdot \cos \omega t \cdot \frac{1}{\cos \varphi} \cdot \cos (\alpha+\varepsilon-\varphi)+G \cdot \frac{1}{\cos \varphi} \cdot \sin (\alpha-\varphi)
$$

Downward condition of the plant fragment on the sifter is given by the following equation:

$$
m \cdot a_{c}=\frac{G}{g} \cdot \omega^{2} \cdot A \cdot \cos \omega t \cdot \frac{1}{\cos \varphi} \cdot \cos (\alpha+\varepsilon-\varphi)+\frac{G}{\cos \varphi} \cdot \sin (\alpha-\varphi) \geq 0
$$

If we choose as movement plane the plane perpendicular to the sifter which contains the force $\mathrm{F}$, the movement equation will be:

$$
\ddot{x}_{c}=\omega^{2} \cdot A \cdot \cos \omega t \cdot \frac{\cos (\alpha+\varepsilon-\varphi)}{\cos \varphi}+g \cdot \frac{\sin (\alpha-\varphi)}{\cos \varphi}
$$


A first integration, with an integration constant $c_{1}$, expresses the velocity field:

$$
\ddot{x}_{c}=-\omega \cdot A \cdot \sin \omega t \cdot \frac{\cos (\alpha+\varepsilon-\varphi)}{\cos \varphi}+g \cdot \frac{\sin (\alpha-\varphi)}{\cos \varphi} \cdot t+c_{1}
$$

Another integration with an integration constant $c_{2}$ leads to the field of coordinates:

$$
x_{c}=-A \cdot \cos \omega t \cdot \frac{\cos (\alpha+\varepsilon-\varphi)}{\cos \varphi}+\frac{g}{2} \cdot \frac{\sin (\alpha-\varphi)}{\cos \varphi} \cdot t^{2}+c_{1} \cdot t+c_{2}
$$

In the plane of sifters movement on direction $\mathrm{Oy}$, the movement equation is:

$$
\ddot{y}_{c}=0
$$

By double integration of equation (11), with constants $\mathrm{c}_{3}$ and $\mathrm{c}_{4}$, implies:

$$
y_{c}=c_{3} \cdot t+c_{4}
$$

Trajectory equation of the fragment is obtained by eliminating the time in equations (12) and (10):

$$
\begin{aligned}
& x_{c}=-A \cdot \cos \left[\omega\left(\frac{y_{c}-c_{4}}{c_{3}}\right)\right] \cdot \frac{\cos (\alpha+\varepsilon-\varphi)}{\cos \varphi}+\frac{g}{2} \cdot \frac{\sin (\alpha-\varphi)}{\cos \varphi} \cdot\left(\frac{y_{c}-c_{4}}{c_{3}}\right)^{2} \\
& +c_{1} \cdot\left(\frac{y_{c}-c_{4}}{c_{3}}\right)+c_{2}
\end{aligned}
$$

On a plant fragment going up on an oscillatory plane surface will act the forces presented in Figure $2 \mathrm{~b}$.

In this case, the movement equation of the fragment is given by:

$$
m \cdot \frac{d v}{d t}=F \cdot \cos (\alpha+\varepsilon)-G \cdot \sin \alpha-F_{f}
$$

where:

$$
\begin{aligned}
N & =G \cdot \cos \alpha+F \cdot \sin (\alpha+\varepsilon) \\
F_{f} & =\mu \cdot N=\operatorname{tg} \varphi \cdot[G \cdot \cos \alpha+F \cdot \sin (\alpha+\varepsilon)]
\end{aligned}
$$

By replacing all terms in equation 14 we obtain:

For

$$
\begin{aligned}
& m \cdot a=F \cdot \cos (\alpha+\varepsilon)-G \cdot \sin \alpha-F_{f}= \\
& F \cdot \cos (\alpha+\varepsilon)-G \cdot \sin \alpha-\operatorname{tg} \varphi \cdot[G \cdot \cos \alpha+F \cdot \sin (\alpha+\varepsilon)]
\end{aligned}
$$

$$
F=\frac{G}{g} \omega^{2} \cdot A \cdot \cos \omega t
$$

It results: 


$$
\begin{aligned}
& m \cdot a_{n}=\frac{G}{g} \omega^{2} \cdot A \cdot \cos \omega t \cdot[\cos (\alpha+\varepsilon)-\operatorname{tg} \varphi \cdot \sin (\alpha+\varepsilon)]-G \cdot(\cos \alpha+\operatorname{tg} \varphi \cdot \cos \alpha)= \\
& \frac{G}{g} \cdot \frac{\omega^{2} \cdot A \cdot \cos \omega t}{\cos \varphi} \cdot \cos (\alpha+\varepsilon+\varphi)-G(\sin \alpha+\varphi) \cdot \frac{1}{\cos \varphi}
\end{aligned}
$$

It is found that:

$$
a_{n}=\frac{\omega^{2} \cdot \cos \omega t}{\cos \varphi} \cdot \cos (\alpha+\varepsilon+\varphi)-g \cdot \sin (\alpha+\varphi) \cdot \frac{1}{\cos \varphi}
$$

The upwarding condition of plant fragment on the sifter is:

$$
a_{n} \geq 0
$$

If we choose as movement plane the plane perpendicular to the sifter which contains the force $\mathrm{F}$, the movement equation will be:

$$
\ddot{x}_{n}=\omega^{2} \cdot A \cdot \cos \omega t \cdot \frac{\cos (\alpha+\varepsilon+\varphi)}{\cos \varphi}-g \cdot \frac{\sin (\alpha+\varphi)}{\cos \varphi}
$$

A first integration, with an integration constant $\mathrm{c}^{{ }_{1}}{ }_{1}$, describes the velocity field:

$$
\ddot{x}_{n}=-\omega \cdot A \cdot \sin \omega t \cdot \frac{\cos (\alpha+\varepsilon+\varphi)}{\cos \varphi}-g \cdot \frac{\sin (\alpha+\varphi)}{\cos \varphi} \cdot t+c_{1}^{\prime}
$$

A new integration, with an integration constant $c^{\prime}{ }_{2}$, leads to the coordinates field:

$$
x_{n}=-A \cdot \cos \omega t \cdot \frac{\cos (\alpha+\varepsilon+\varphi)}{\cos \varphi}-\frac{g}{2} \cdot \frac{\sin (\alpha+\varphi)}{\cos \varphi} \cdot t^{2}+c_{1}^{\prime} \cdot t+c_{2}^{\prime}
$$

In the plane of sifters movement on direction $\mathrm{Oy}$, the movement equation is:

$$
\ddot{y}_{n}=0
$$

By double integration of equation (25), with constants $\mathrm{c}_{3} \mathrm{si}^{\prime}{ }_{4}$, implies:

$$
y_{n}=c_{3}^{\prime} \cdot t+c_{4}^{\prime}
$$

Trajectory equation of the fragment is obtained by eliminating the time in equations (26) and (24):

$$
x_{n}=-A \cdot \cos \left[\omega\left(\frac{y_{n}-c_{4}^{\prime}}{c_{3}^{\prime}}\right)\right] \cdot \frac{\cos (\alpha+\varepsilon+\varphi)}{\cos \varphi}-\frac{g}{2} \cdot \frac{\sin (\alpha+\varphi)}{\cos \varphi} \cdot\left(\frac{y_{n}-c_{4}^{\prime}}{c_{3}^{\prime}}\right)+c_{2}^{\prime}
$$

\section{b) Relative movement through jumps of a plant fragment on vibratory plane sifter}

After separation, the fragment is thrown by the sifter with initial velocity $\mathrm{v}_{\mathrm{i}}$, equal to the velocity $\mathrm{v}_{\mathrm{s}}$ of the sifter, at the time of separation. After its throwing, the fragment has a parabolic trajectory, reaching back to the sifter, as presented in Figure 3 [7]. 


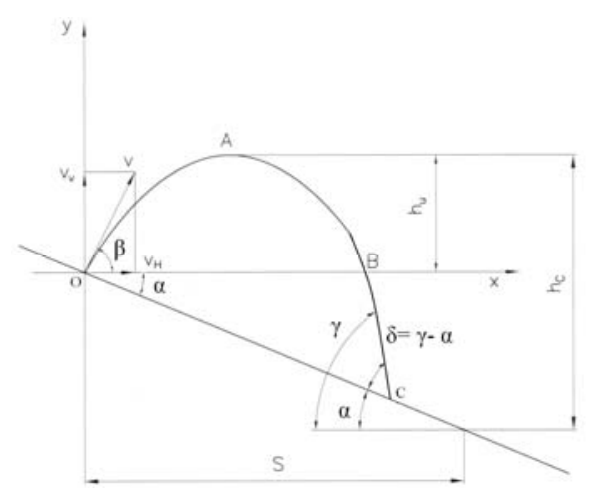

Fig. 3. The parabolic trajectory of the vegetal fragment, reaching back to the sifter.

Initial velocity of the fragment is:

$$
v_{i}=r \cdot \omega \cdot \sin \omega t_{0}
$$

where $t_{0}$, is the time of fragment separation, given as:

$$
t_{0}=\frac{1}{\omega} \cdot \arccos \left(\frac{g}{r \cdot \omega^{2}} \cdot \frac{\cos \alpha}{\sin \gamma}\right)=\frac{1}{\omega} \cdot \arccos \frac{1}{C_{a}}
$$

Since the initial acceleration, $\mathrm{a}_{\mathrm{i}}$, is:

$$
\begin{aligned}
& a_{i}=r \omega^{2} \cdot \cos \omega t_{0} \\
& \cos \omega t_{0}=\frac{g}{r \cdot \omega^{2}} \cdot \frac{\cos \alpha}{\sin \gamma}=\frac{1}{C_{a}}
\end{aligned}
$$

Using equations (29) and (31), the following equation is obtained for the initial velocity of the fragment:

$$
v_{i}=r \cdot \omega \cdot \sqrt{1-\frac{1}{C_{a}^{2}}}
$$

According to Figure 3, the parametric equations describing the trajectory of fragment jump are:

$$
\begin{aligned}
& x=v_{i} \cdot \cos \beta \cdot t \\
& y=v_{i} \cdot \sin \beta \cdot t-\frac{g \cdot t^{2}}{2}
\end{aligned}
$$

By eliminating the time in equations (33) and (34), the equation of fragment trajectory is obtained:

$$
y=x \cdot \operatorname{tg} \beta-\frac{g}{2 \cdot v_{H}^{2}} \cdot x^{2}
$$

Where, for the horizontal component of initial velocity, was used the following notation:

$$
\mathrm{v}_{\mathrm{H}}=\mathrm{v} \cos \beta
$$


Next are determined the coordinates of characteristic points (A, B, C) of the fragment jump trajectory:

- Coordinates of point A;

As it can be noticed in Figure 3, point A is the maximum point of fragments trajectory function (equation (35)), hence:

$$
\frac{d y}{d x}=\operatorname{tg} \beta-\frac{g}{v_{H}^{2}} \cdot x=0
$$

It results:

$$
\left\{\begin{array}{l}
x_{A}=\frac{v_{H}^{2}}{g} \cdot \operatorname{tg} \beta \\
y_{A}=\frac{v_{H}^{2}}{2 \cdot g} \cdot \operatorname{tg}^{2} \beta
\end{array}\right.
$$

- Coordinates of point B;

Since the ordinate of point $\mathrm{B}$ is $\mathrm{y}_{\mathrm{B}}=0$, from the equation describing the trajectory of the fragment (equation (35)), is obtained its abscissa:

$$
x_{B}=\frac{2 \cdot v_{H}^{2}}{g} \cdot \operatorname{tg} \beta
$$

- Coordinates of point $\mathrm{C}$;

Point $\mathrm{C}$ represents the falling of the fragment on the sifter, so it results from the intersection of fragments trajectory with the track of sifters plane. The track of sifter plane has the following equation:

$$
y=-x \cdot \operatorname{tg} \alpha
$$

Thus, by solving the system formed by equations (35) and (40), it results:

$$
\left\{\begin{array}{l}
x_{C}=\frac{2 \cdot v_{H}^{2}}{g} \cdot(\operatorname{tg} \beta+\operatorname{tg} \alpha) \\
y_{C}=-\frac{2 \cdot v_{H}^{2}}{2} \cdot(\operatorname{tg} \beta+\operatorname{tg} \alpha) \cdot \operatorname{tg} \alpha
\end{array}\right.
$$

- length of fragment jump along the sifter is:

$$
S_{0}=\frac{x_{C}}{\cos \alpha}=\frac{2 \cdot v_{H}^{2}}{g} \cdot \frac{\operatorname{tg} \beta+\operatorname{tg} \alpha}{\cos \alpha}=\frac{2 \cdot v_{H}^{2}}{g} \cdot \frac{\sin \gamma}{\cos \beta \cdot \cos ^{2} \alpha}
$$

- Falling angle of the fragment towards the sifter

The angle between the tangent to the trajectory at its current point and the x-axis is given by: 


$$
\operatorname{tg} \gamma=\frac{d y}{d x}=\operatorname{tg} \beta-\frac{g}{v_{H}^{2}} \cdot x
$$

For falling point $\mathrm{C}$ of the fragment on the sifter $\mathrm{x}=\mathrm{x}_{\mathrm{C}}, \mathrm{x}_{\mathrm{C}}$ being defined by equation (41), from equation (43), it results:

$$
\operatorname{tg} \gamma=-(\operatorname{tg} \beta+2 \cdot \operatorname{tg} \alpha)
$$

The angle between the tangent to the trajectory at point $\mathrm{C}$ and the sifter (falling angle of the fragment on the sifter) is:

$$
\delta=\gamma-\alpha
$$

- Time of fragment jump is:

$$
t_{s}=t_{u}+t_{c}
$$

where:

$t_{u}$ is the time in which the fragment moves along portion OA of the trajectory;

$t_{c}$ is the time in which the fragment moves along portion AC.

Time $t_{u}$ is given by:

$$
t_{u}=\sqrt{\frac{2 \cdot h_{u}}{g}}=\sqrt{\frac{2 \cdot y_{A}}{g}}=\frac{v_{H}}{g} \cdot \operatorname{tg} \beta
$$

Time $t_{c}$ is given by:

$$
t_{c}=\sqrt{\frac{2 \cdot h_{c}}{g}}
$$

where $h_{c}$, taking into account equations (38) and (41), is:

$$
h_{c}=y_{A}+\left|y_{C}\right|=\frac{v_{H}^{2}}{g} \cdot\left[\frac{\operatorname{tg}^{2} \beta}{2}+2 \cdot(\operatorname{tg} \beta+\operatorname{tg} \alpha) \cdot \operatorname{tg} \alpha\right]
$$

With equation (30), equation (31) becomes:

$$
t_{c}=\frac{v_{H}}{g} \cdot(\operatorname{tg} \beta+2 \cdot \operatorname{tg} \alpha)
$$

Taking into account equations (32) and (50), it results:

$$
t_{s}=t_{u}+t_{c}=\frac{2 \cdot v_{H}}{g} \cdot(\operatorname{tg} \beta+\operatorname{tg} \alpha)
$$

Using equations (32) and (36), equation (51) becomes: 


$$
t_{s}=\frac{2 \cdot v_{i}}{g} \cdot \frac{\sin \gamma}{\cos \alpha}=2 \cdot \frac{r \cdot \omega}{g} \cdot \frac{\sin \gamma}{\cos \alpha} \cdot \sqrt{1-\frac{1}{C_{a}^{2}}}=\frac{2}{\omega} \cdot \sqrt{C_{a}^{2}-1}
$$

- Height of fragment jump towards the sifter, h.

In order to determine this parameter, we consider the trajectory of the fragment depending on system XOY, in Figure 4, whose OY axis is directed along the sifter. The connection between the coordinates of the considered point and each of the mentioned coordinate systems, is:

$$
\left\{\begin{array}{l}
x=X \cdot \cos \alpha+Y \cdot \sin \alpha \\
y=-X \cdot \sin \alpha+Y \cdot \cos \alpha
\end{array}\right.
$$

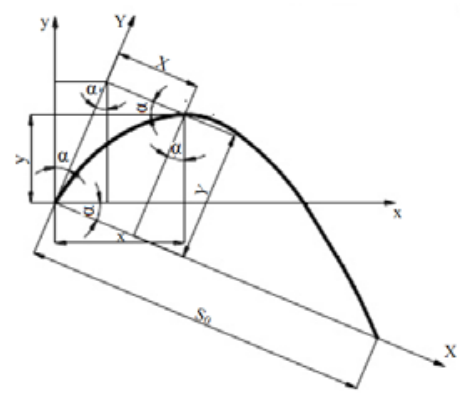

Fig. 4. The trajectory of the fragment.

Taking into account equation (53), equation (35) of fragment trajectory of fragment becomes:

$$
(\cos \alpha \cdot \operatorname{tg} \beta+\sin \alpha) \cdot x+(\sin \alpha \cdot \operatorname{tg} \beta-\cos \alpha) \cdot y \cdot \frac{g}{2 \cdot v_{H}^{2}} \cdot(x \cdot \cos \alpha+y \cdot \sin \alpha)^{2}=0
$$

It results the trajectory equation of fragment jump related to the new system of coordinates XOY.

For $\mathrm{Y}=0$, is obtained the trajectory equation of fragment jump:

$$
X=\frac{2 \cdot v_{H}^{2}}{g} \cdot \frac{\operatorname{tg} \beta+\operatorname{tg} \alpha}{\cos \alpha}=S_{0}
$$

Hence the lenght of fragment jump towards the sifter.

Next, to determine the height of fragment jump towards the sifter, relations (53) become:

$$
\left\{\begin{array}{l}
x \cdot \sin \alpha=X \cdot \sin \alpha \cdot \cos \alpha+Y \cdot \sin ^{2} \alpha \\
y \cdot \cos \alpha=-X \cdot \sin \alpha \cdot \cos \alpha+Y \cdot \cos ^{2} \alpha
\end{array}\right.
$$

Of which it results:

$$
\mathrm{Y}=\mathrm{x} \cdot \sin \alpha+\mathrm{y} \cdot \cos \alpha
$$

If equation (35) of fragment trajectory is taken into account, equation (57) becomes:

$$
Y=x \cdot(\sin \alpha+\operatorname{tg} \beta \cdot \cos \alpha)-\frac{g}{2 \cdot v_{H}^{2}} \cdot \cos \alpha \cdot x^{2}
$$


By canceling the derivate of function (58) with respect to $\mathrm{x}$, it results:

$$
\frac{d Y}{d x}=\sin \alpha+\operatorname{tg} \beta \cdot \cos \alpha-\frac{g}{2 \cdot v_{H}^{2}} \cdot \cos \alpha \cdot x
$$

Thus:

$$
x=\frac{\sin \alpha+\operatorname{tg} \beta \cdot \cos \alpha}{\cos \alpha} \cdot \frac{v_{H}^{2}}{g}=\frac{\sin \gamma}{\cos \beta \cdot \cos \alpha} \cdot \frac{v_{H}^{2}}{g}
$$

For this parameter, from equation (58), it results:

$$
Y_{\max }=\frac{\sin (\beta+\alpha)}{\cos \beta \cdot \cos \alpha} \cdot \frac{v_{H}^{2}}{g}=\frac{\sin \gamma}{\cos \beta \cdot \cos \alpha} \cdot \frac{v_{H}^{2}}{2 \cdot g}=h_{0}
$$

\section{RESULTS}

Case studies. Numerical application. Based on the theoretical study of the literature on the kinematic regime of sifters and knowing some of the constructive and functional parameters of plant sorter, was made a theoretical calculation, which characterizes the behavior of plant fragments in the sorting process on plane sifters with longitudinal oscillations.

Knowing the parameters of the vibratory regime of the plant sorter was theoretically calculated the trajectory of a plant fragment on the surface of the sifter, whose tilting angle $\alpha$ with the horizontal, $\alpha$, can have three values: $\alpha=13.33^{\circ}, 12.08^{0}, 14.7^{\circ}$. Using a numerical calculation program in Mathcad and the equations from the study of trajectory were calculated some parameters of the trajectory of fragment jump on the surface of the sifter, and the obtained values are presented in Table 1.

After its separation from the sifter, the fragment is thrown with initial velocity $\mathrm{v}_{\mathrm{i}}$, equal to the velocity of sifter at the time of separation, corresponding to time $t_{0}$, hence the time of fragment separation from the sifter. The initial velocity of plant fragment and the corresponding time of its separation from the sifter have the same value for the three tilting angles, and they vary with the frequency, as it can be seen in Figure 5.

From the analysis of Figure 5, it is found that the initial velocity of the fragment increases with frequency increase and the time to fragment separation from the sifter decreases with frequency oscillations.

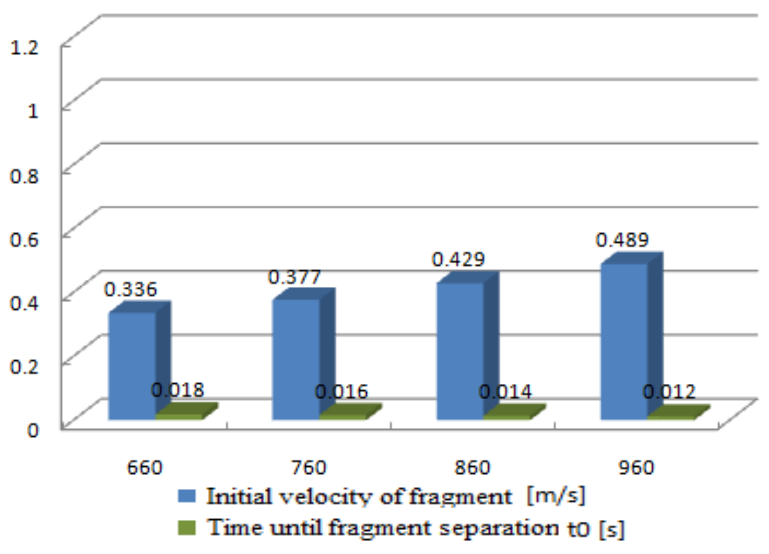

Fig. 5. The initial velocity of plant fragment and the corresponding time of its separation from the sifter.

Using the equation of the length of fragment jump along the sifter, were determined the angle of jump ( $\beta$ ) and the angle of fragment trajectory with the horizontal plane $(\gamma)$ for the three tilting angles of the sifter. 
Table 1. Calculated some parameters of the trajectory of fragment jump on the surface of the sifter.

\begin{tabular}{|c|c|c|c|c|c|}
\hline $\begin{array}{l}\text { Tilting } \\
\text { angle of } \\
\text { the sifter } \\
(\alpha)\left[^{0}\right]\end{array}$ & $\begin{array}{l}\text { Fragment } \\
\text { jump angle } \\
(\beta)\left[^{0}\right]\end{array}$ & $\begin{array}{c}\text { Plant fragment } \\
\text { length of jump } \\
\text { towards the } \\
\text { sifter } \\
\text { S [mm }]\end{array}$ & $\begin{array}{c}\text { Mean } \\
\text { forwarding } \\
\text { velocity of } \\
\text { plant fragment } \\
\text { along the sifter } \\
\mathrm{v}_{\mathrm{H}}[\mathrm{m} / \mathrm{s}]\end{array}$ & $\begin{array}{c}\text { Fragment } \\
\text { trajectory angle } \\
\text { with the } \\
\text { horizontal plane } \\
(\gamma)\left[^{0}\right]\end{array}$ & $\begin{array}{l}\text { Height of } \\
\text { fragment jump } \\
\text { towards the } \\
\text { sifter } \\
{[\mathrm{mm}]}\end{array}$ \\
\hline \multicolumn{6}{|c|}{$C_{a}=3.58 ; \omega=100.5 \mathrm{~s}^{-1} ; A=3.48 \mathrm{~mm}$} \\
\hline 12.08 & 65.62 & 9.69 & 0.139 & 69.21 & 2.26 \\
\hline 13.33 & 63.14 & 11 & 0.152 & 67.78 & 2.47 \\
\hline 14.7 & 60.42 & 12 & 0.166 & 66.37 & 2.68 \\
\hline \multicolumn{6}{|c|}{$C_{a}=3.6 ; \omega=90 \mathrm{~s}^{-1} ; A=4.36 \mathrm{~mm}$} \\
\hline 12.08 & 65.84 & 12 & 0.154 & 69.37 & 2.83 \\
\hline 13.33 & 63.34 & 13 & 0.169 & 67.92 & 3.09 \\
\hline 14.7 & 60.60 & 15 & 0.185 & 66.49 & 3.37 \\
\hline \multicolumn{6}{|c|}{$C_{a}=3.62 ; \omega=79.5 \mathrm{~s}^{-1} ; A=5.62 \mathrm{~mm}$} \\
\hline 12.08 & 65.78 & 16 & 0.176 & 69.33 & 3.68 \\
\hline 13.33 & 63.28 & 17 & 0.193 & 67.88 & 4.02 \\
\hline 14.7 & 60.55 & 19 & 0.211 & 66.46 & 4.37 \\
\hline \multicolumn{6}{|c|}{$C_{a}=3.58 ; \omega=69 \mathrm{~s}^{-1} ; A=7.38 \mathrm{~mm}$} \\
\hline 12.08 & 65.71 & 20 & 0.201 & 69.28 & 4.79 \\
\hline 13.33 & 63.22 & 23 & 0.220 & 67.84 & 5.22 \\
\hline 14.7 & 60.49 & 25 & 0.241 & 66.42 & 5.68 \\
\hline
\end{tabular}

Depending on the frequency values, in Figure 6 was varied the position of the fragment at the time of its separation from the sifter and after the separation. From the graph, it can be observed that the angles do not vary significantly with the increase of frequency oscillation.

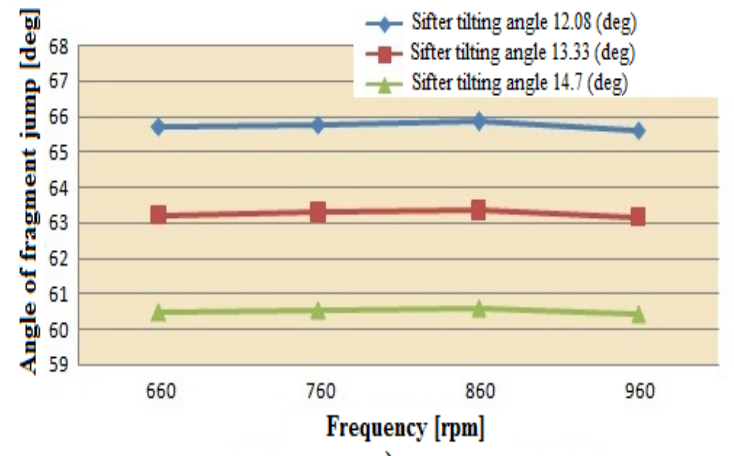

a)

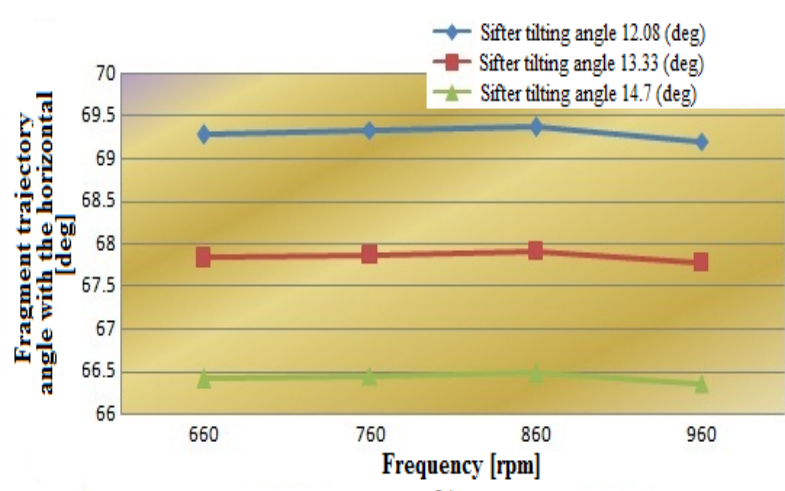

b)

Fig. 6. Variation of position of the fragment at the time of its separation from the sifter and after the separation.

Given the length and height of fragment jump for the considered tilting angles, in Figure 7 it can be observed that their values decrease with increasing frequency. For example, at the rotation frequency of $960 \mathrm{rpm}$, the maximum length is between $10 \div 12 \mathrm{~mm}$ depending on the sifter angle and the height of jump vary between 2.26 and $2.68 \mathrm{~mm}$. 


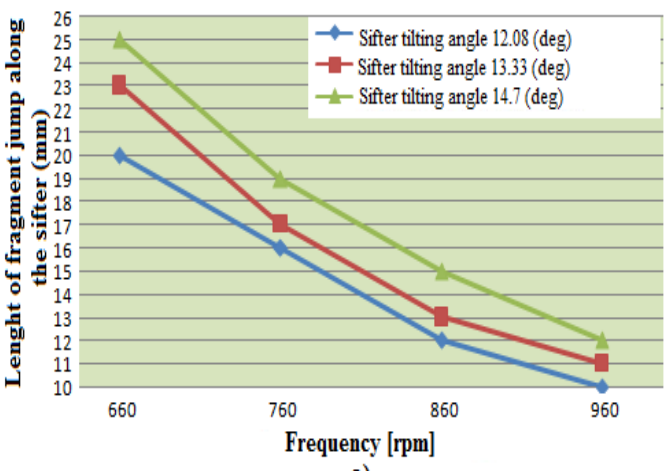

a)

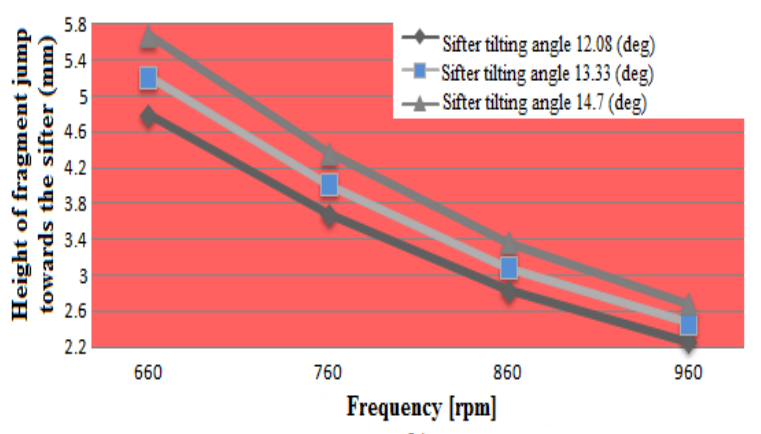

b)

Fig. 7. The length and height of fragment jump for the considered angles.

In terms of time until fragment separation from the sifter and the time of jump during the trajectory were plotted the graphs in Figure 8. It was found that both times decrease with the increase of frequency.

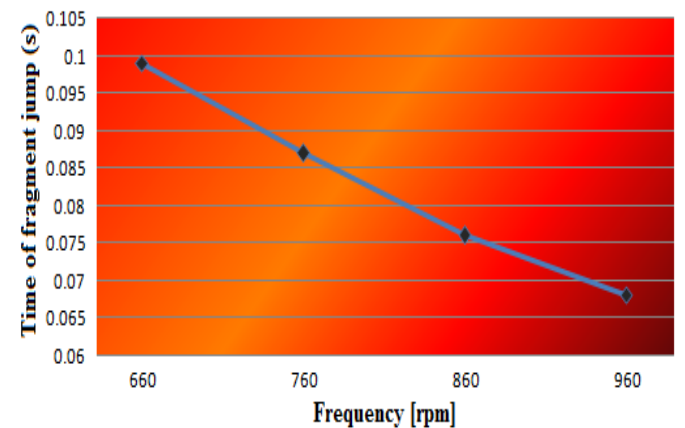

a)

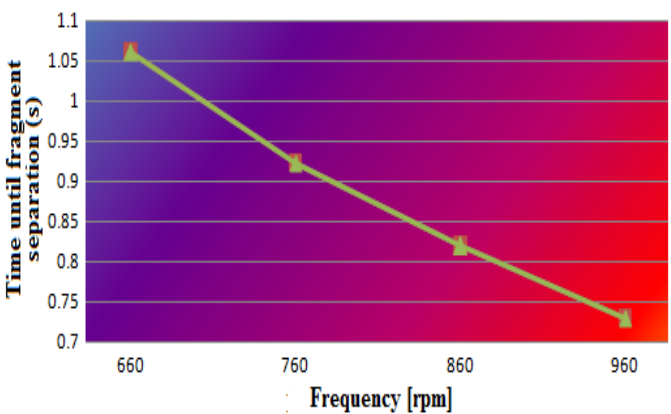

b)

Fig. 8. The time until fragment separation from the sifter and the time of jump during the trajectory.

The forward velocity of the fragment along the sifter, presented in Figure 9, drops to $0.139 \mathrm{~m} / \mathrm{s}$, for the minimum tilting angle of the sifter at a frequency of $960 \mathrm{rpm}$.

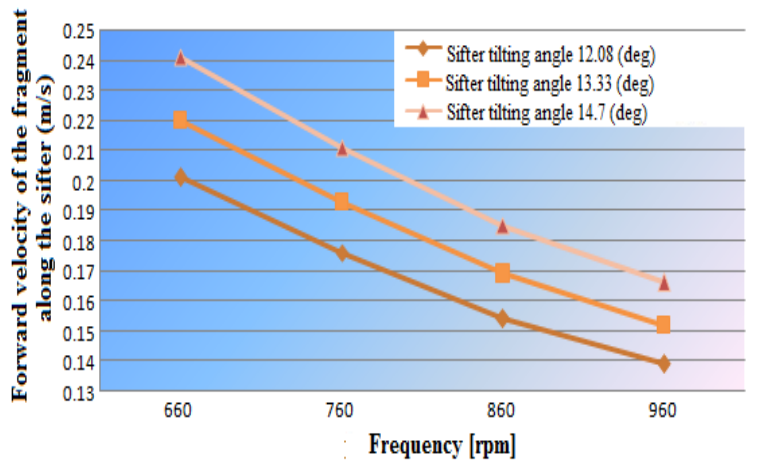

Fig. 9. Velocity of the fragment along the sifter.

Based on the trajectory equation of fragment jump were performed the trajectories for three tilting positions of the plant sorter, depending on the frequency. Trajectories of fragment jump for different frequencies are plotted in Figure 10. It was found that the trajectories of the jump vary, as they distance from the vertical of the release point with decreasing of the oscillation frequency, so fragment jump is longer when the frequency decreases and the tilting angle increases. 

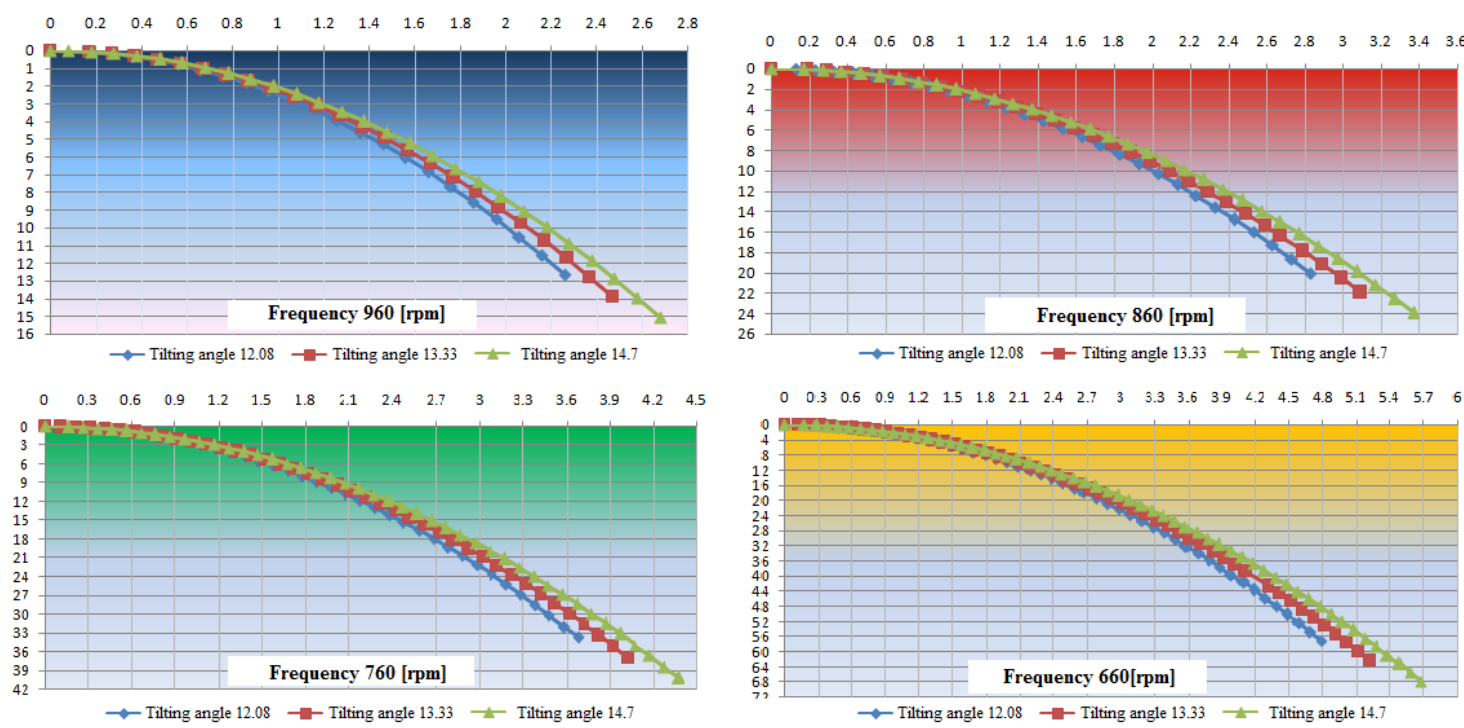

Fig. 10. Trajectories of fragment jump for different frequencies.

\section{CONCLUSIONS}

In this paper, there were theoretically established the movement equations for determining the place where vegetal fragments detach from the plane sifter, the speed with which the fragment detaches and fragment trajectory during its jump against the sifter.

Following the analysis of case study, have been found the following:

- the frequency of oscillation of sifter influences the initial speed of fragment and duration of fragment jump in its trajectory;

- the fragment position when it detaches and after its detachment from the sifter, jump length and height, speed of fragment along the sifter, all are influenced by both oscillation frequency and sifter tilt angle;

- the value of fragment speed during the jump against the sifter is significantly influenced by sifter tilting angle variation, when it ranges between $12-15^{\circ}$ and also by the oscillation frequency of 660-960 osc/min;

- higher the sifter tilting angle for different oscillation frequencies is, higher becomes the fragment speed along the sifter;

- analysis of jump parameters has shown significant increase for the fragment jump length in comparison with sifter from 9.7 up to $25 \mathrm{~mm}$ and less important increase for jump height with values between 2.2$5.6 \mathrm{~mm}$;

- analysis of fragment jump trajectories comparatively traced has emphasized that fragment jump is longer when the frequency is lower and sifter tilting angle increases.

In conclusion, in order to obtain an efficient separation, the jump parameters must be according to the size of initial speed components, namely:

- small values of displacement speed of fragments on the sifter and length and height of fragment jump determine an appropriate sorting process, but the sifter working capacity is reduced; theoretical data obtained by calculation have emphasized the fact that for sifter tilting angle of $12.08^{0}$, along with sifter oscillation frequency growth, respectively the kinematic angle, the fragments displacement speed on sifter increases, framing between the practical values $(0.08-0.12 \mathrm{~m})$, recommended by specialty literature;

- high values of fragment displacement speed on sifter and of length and height of fragment jump are not recommended, as they inappropriately influence the process of separation of fragments, a big part of unseparated fragments remaining. So, the sieved product will be of low quality.

Theoretical results obtained from numerical analysis and adopted for the parameters of classifier of medicinal plants are going to be experimentally checked, in order to improve the working regime. 
A comparative presentation of values obtained by means of the mathematical model and of those able to be experimentally obtained is very important for the optimization of working process of plane sifter classifiers and also for the experts in the field.

\section{REFERENCES}

[1] Florea, C.C., Issues Regarding Quality Control Of The Medicinal Plants Raw Materials, in Book of Abstracts Etnopharmacology Interface Between Bio Foods and Phytomedicines, Transilvania University Press, 2011, p. 76.

[2] Segarceanu, M., Casandroiu, T., Contributii la studiul miscarii semintelor pe suprafata sitelor plane, Studii si cercetari de mecanica agricola, vol. II, 1968.

[3] Krasnicenko, A.V., Manualul constructorului de masini agricole (traducere din limba rusa), vol. II, Editura Tehnica, Bucuresti, 1964.

[4] Iordache, Gh., Masini si utilaje pentru industria alimentara, Ed. Matrix Rom, Bucuresti, 2004.

[5] Tenu, I., Tehnologii, masini si instalatii pentru industrializarea produselor vegetale, Partea a II-a, Curatirea spalarea si conditionarea, Ed. Junimea, Iasi, 1999.

[6] Secara, E., Constantin, F., Mecanica si aplicatiile ei tehnice, Ed. Universitatii Transilvania, Brasov, 2011.

[7] Ene, Gh., Echipamente pentru clasarea si sortarea materialelor solide polidisperse, Ed. Matrix Rom, Bucuresti, 2005.

[8] Bontas, O., Nedeff, V., Mosnegutu, E.F., Panainte, M., Tirtoaca I., Behaviour of solid particles on a flat oscillating surface, Environmental Engineering and Management Journal, vol. 12, no. 1, 2013, p. 17-22.

[9] Mosnegutu, E., Nedeff, V., Panainte-Lehadus, M., Bontas, O., Barsan, N,, Tomozei, C., Chitimus, D., Studies and research concerning the displacement of solid particle on an oscillatory flat surface. Applied Mechanics and materials, vol. 10, 2014.

[10] Zhao, L., Liu, C., Yan, J., A virtual experiment showing single particle motion on a linearly vibrating screen-deck, Mining Science and Technology, vol. 20, 2010, p. 276-280.

[11] Serdar, O., Milan, M., Medicinal and aromatic crops: harvesting, drying and processing, Editura Haworth Press, United States and Canada, 2007.

[12] Xiao, J., Tong, X., Characteristics and efficiency of a new vibrating screen with a swing trace, Particuology, vol. 11, 2013, p. 601-606.

[13] Wang, G., Tong, X., Screening efficiency and screen length of a linear vibrating screen using DEM 3D simulation, Mining Science and Technology (China), vol. 21, 2011, p. 451-455.

[14] Chen, Y., Tong, X., Modeling screening efficiency with vibrational parameters based on DEM 3D simulation, Mining Science and Technology, vol. 20, 2010, p. 615-620.

[15] Raport, D. C., A new mechanism for granular segregation, Phys Rev E 64, 2001, 061304; (http://arxiv.org/abs/cond-mat/0101176v2).

[16]. Casandroiu, T, Utilaje pentru prelucrarea primara si pastrarea produselor agricole, curs 1 Notiuni generale. Procese si utilaje pentru curatirea si sortarea semintelor, Universitatea Politehnica, Facultatea de Mecanica Agricola, Bucuresti, 1993.

[17] INMA, Cartea tehnica a sortatorului de plante medicinale tocate, Bucuresti.

[18] Ene, Gh., Marin, C., Calculul si constructia masinilor vibratoare, Ed. Printech, Bucuresti, 2009. 